\title{
The LOFAR Phased Array Telescope System
}

André W. Gunst, ASTRON, Dwingeloo, the Netherlands, gunst@astron.nl

Mark J. Bentum, ASTRON, Dwingeloo, the Netherlands, bentum@astron.nl

\begin{abstract}
The Low Frequency Array (LOFAR) is the largest telescope in the world operating at a frequency range from 30 to $240 \mathrm{MHz}$. LOFAR is the first radio telescope of its size which uses phased array principles to detect radio signals. More than 10,000 antennas are installed in the field. The antennas are grouped in 44 stations. The maximal distance between the stations is about $1500 \mathrm{~km}$ resulting in a tremendous spatial resolution. In this paper the LOFAR system architecture is discussed and the status is described.
\end{abstract}

\section{INTRODUCTION}

The technical requirements of LOFAR have been driven by a broad and diverse range of scientific goals [1]. Amongst others, these goals include wide-field, high dynamic range imaging, near real-time detection of radio transients, high accuracy pulsar timing, solar monitoring, and radio pulses generated by cosmic ray air showers. To achieve such a wide spectrum of scientific requirements, the system has been designed and built to be both highly flexible as well as extensible [2]. This is achieved by using many small antennas instead of dishes, which are digitized at an early stage in the signal chain. Doing so, an operator is able to point the telescope simultaneously in multiple directions and schedule multiple observations at the same time increasing the efficiency of the telescope. Furthermore part of the flexibility is realized by using custom off the shelf computing hardware for the central systems where all data of the fields is combined. In this way imaging observations can be mixed or followed with other types of observations easily.

In this paper the LOFAR system will be discussed. In Section 2 the geometry of LOFAR is presented. The system architecture is described in section 3. The station subsystem and the Central Systems Architecture (CEP) are discussed in more detail in Sections 4 and 5. The paper concludes with the current status of LOFAR and conclusions.

\section{INSTRUMENT GEOMETRY}

For LOFAR, the sensors should be distributed over a large area to achieve an angular resolution of arcsec accuracy with an acceptable UV coverage. All data coming from the sensors should come together in the correlator. So, on the one hand the instrument should be distributed over a large area, while on the other hand all data should come together in a central location. To balance the hardware and operational costs between (1) the equipment in the field, (2) the transport network and (3) the volume of the central systems, multiple antennas are grouped in so called stations. Within such a station the information of all individual antennas is weighted and summed. Such an array of antennas is also called a phased array. By using this technique a spatial selection on the sky is made, which reduces the instantaneous Field Of View (FOV) of each station. Beams formed at the stations will reduce the total bandwidth requirement on long distance connections to the central processor. For receptors near the central concentrator node (where all the data is coming together), this constraint is relaxed. Within a diameter of $\sim 2 \mathrm{~km}$, signals from each station have a bandwidth which is twice the bandwidth of the remote stations. This inner region is referred to as the core of LOFAR.

A total of 44 stations are built along five spiral arms. The sensor fields are centrally condensed, following a logarithmic distribution. The configuration is two dimensional in order to yield a good instantaneous UV coverage. Furthermore, the configuration is a balance between imaging capabilities under a variety of observing conditions, infrastructural cost and availability of land. Interest from other countries in Europe was shown and led to an instrument with a much longer baseline than originally anticipated. In Figure 1 the LOFAR configuration including the European stations in Germany, France, Sweden and the $\mathrm{UK}$ is shown.

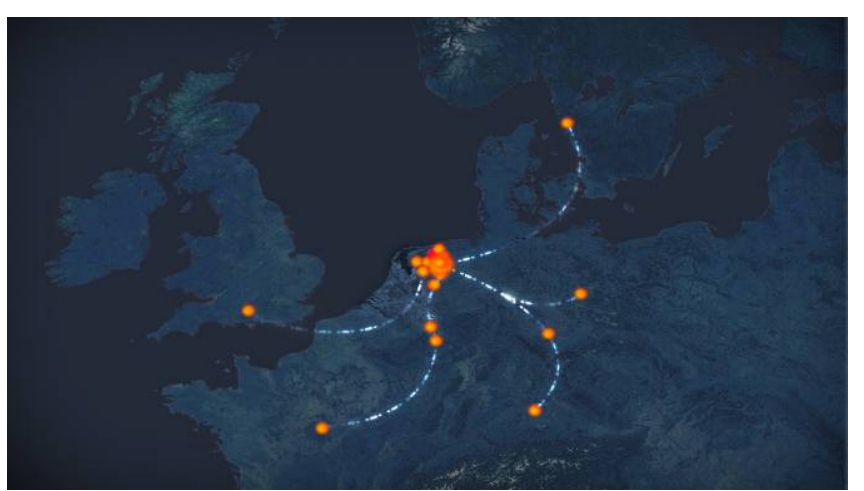

Figure 1 -LOFAR stations layout (picture by Blue Media)

The stations installed in the Netherlands are depicted in Figure 2. Six stations in the core of LOFAR are installed close together on an island, called the superterp, as is shown in Figure 3. 


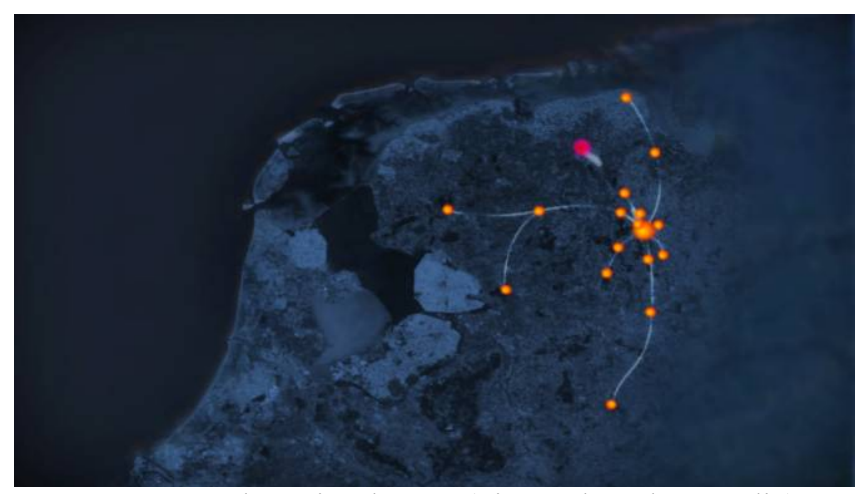

Figure 2 - Dutch station layout (picture by Blue Media)

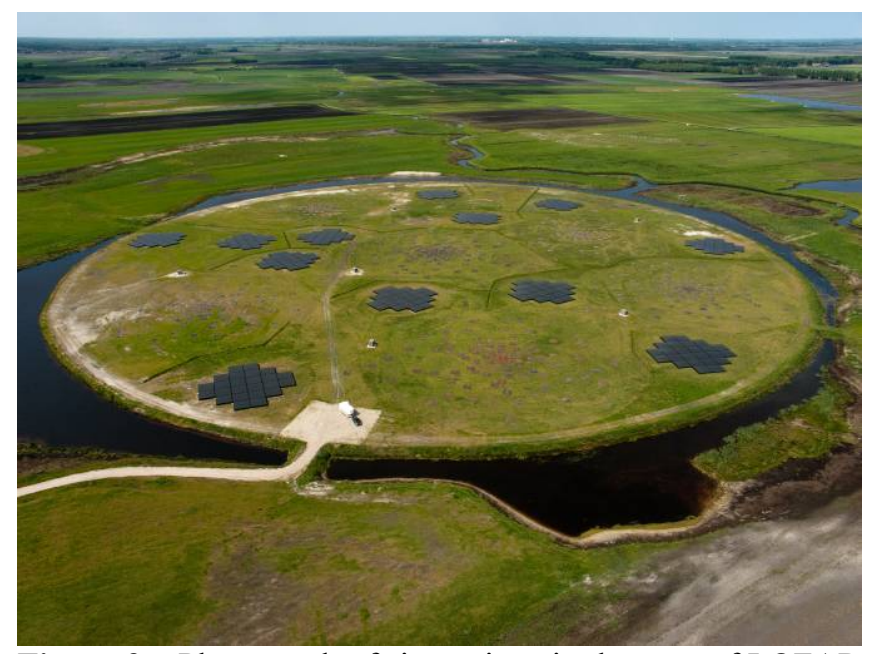

Figure 3 - Photograph of six stations in the core of LOFAR (picture by Top-foto, Assen)

Each Dutch station will comprise of $2 \times 48$ Low Band Antennas (LBA) for the frequency range from 30 to 80 $\mathrm{MHz}$ and 48 High Band Antennas (HBA) for the frequency range from 120 to $240 \mathrm{MHz}$. Both types of antennas are dual polarized. The restriction in the Dutch station is that only 96 signal paths can be used simultaneously. For the LBAs this means one can select only 48 LBA antennas for both polarizations at the same time or for example 96 antennas for one single polarization. This gives extra flexibility in the configuration for modest costs. Two basic LBA configurations are installed in the field: a closed pack array (the inner array) and an outer array in which the LBAs have a larger separation. Since the outer array is sparser, the effective area will be larger at the expense of a smaller field of view (beam size) [3]. Using all the 96 antennas increases evidently the sensitivity and is of use for observations in which the polarization properties of the sources is not relevant. The LBA field diameter is about $85 \mathrm{~m}$. For the antenna configuration of the LBA an irregular array is chosen to scramble the grating lobes. The LOFAR fields are rotated compared to each other to scramble the grating lobes even more. All individual dipoles are back rotated in order to guarantee the same North-South orientation of all dipoles.

The core stations, centered near the small town Exloo, can deliver twice the amount of data than each remote or
International station. Each core station also consists of 96 LBAs and 48 HBAs. The LBA antenna field is identical to the remote stations. In each core station two small HBA fields of each 24 tiles are present as indicated in Figure 4.

A core station can be split up in two core stations resulting in much more baselines. Furthermore the field of view of each core station for the individual HBA field is larger.

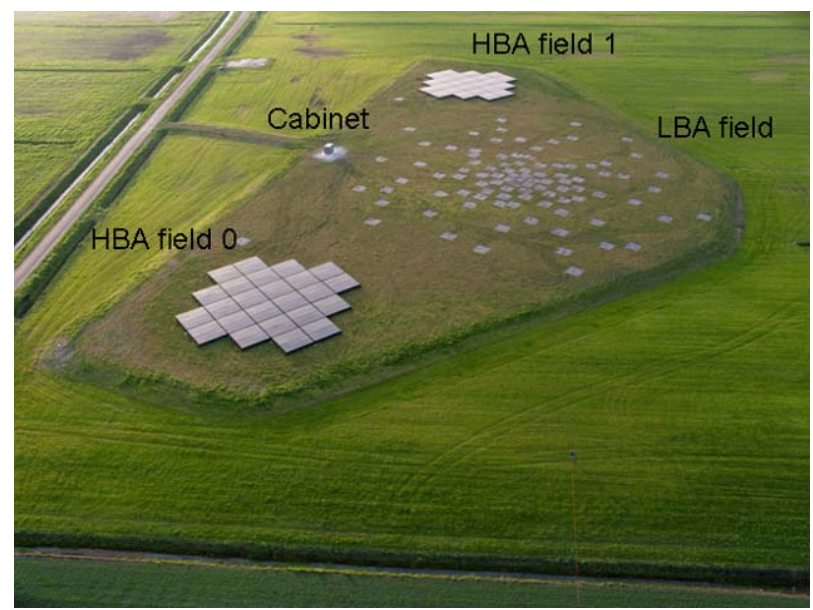

Figure 4 - Photograph of core station layout (picture by Top-foto, Assen)

The International stations in LOFAR are twice as large as the Dutch stations, offering more effective area on the longer baselines. An International station has 96 LBAs and 96 HBA tiles.

\section{SYSTEM ARCHITECTURE}

LOFAR will be the first large radio telescope system, wherein a huge amount of small sensors are used to achieve its sensitivity instead of a small number of big dishes. The main reasons for this are:

- For the low frequencies involved in LOFAR traditional telescopes would be very large and hence costly

- Pointing can be done electronically, without using moveable parts and hence saving on maintenance costs

- It enables pointing in multiple directions at the same time

- It provides operational flexibility (e.g. rapid switching between observations is possible)

Since the concept of LOFAR is so different compared with the traditional radio telescopes, the astronomical science that can be done with it is completely different as well.

Despite the new concept the processing steps of a radio telescope remain the same [4]. The general data path for an aperture synthesis array is depicted in Figure 5. 


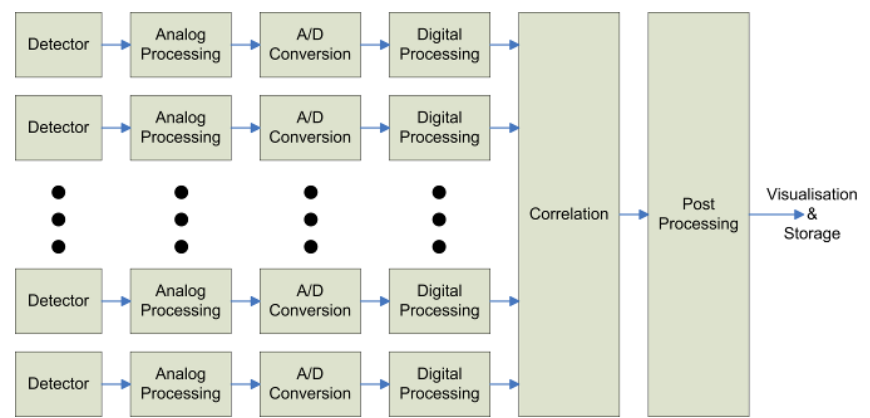

Figure 5 - General datapath of an aperture synthesis array

In LOFAR, the detector is composed of multiple sensors. Since these sensors convert electromagnetic radiation into electronic signals, the sensors are further referred to as antennas. Ideally, the number of detectors equals the number of antennas to accommodate all sky imaging. However, cost of data transport and processing power limits the number of antennas which can be afforded for a feasible design with a reasonable price. The analog processing shown in Figure 5 covers the (low noise) amplification, filtering, analog signal transport and further signal conditioning functions before the signal is converted into the digital domain (Analog/Digital (A/D) conversion block). From there, the signals are digitally conditioned before entering the correlator. Typical operations in LOFAR digital processing are frequency selection, beam forming, delay tracking and fringe stopping. In the correlator, all signals are correlated with each other to form the cross correlation matrix. Furthermore, the correlation results are calibrated for instrumental and environmental effects. Additionally, known sources are subtracted to enhance the dynamic range. Another post processing task is to transform the correlation products into an image.

\section{Architectural Decomposition of the System}

The top level LOFAR system architecture is depicted in Figure 6. Each subsystem is discussed in the next subsections briefly. Since the stations and Central Processing (CEP) subsystems are large and complex hardware intensive systems they are discussed more thoroughly in separate sections.

Stations-In the stations the electromagnetic field received by the station antennas is converted into signals with spatial and spectral sensitivity in the digital domain. The station output is a time series of voltage beams, although intermediate signals can also be made available for specific observations. A station is also able to store the raw antenna data in parallel with the streaming data flow. Hence two types of information flow out of each station: beam data and buffered data. To time synchronize each station a GPS (Global Positioning System) signal is used as input to the station.

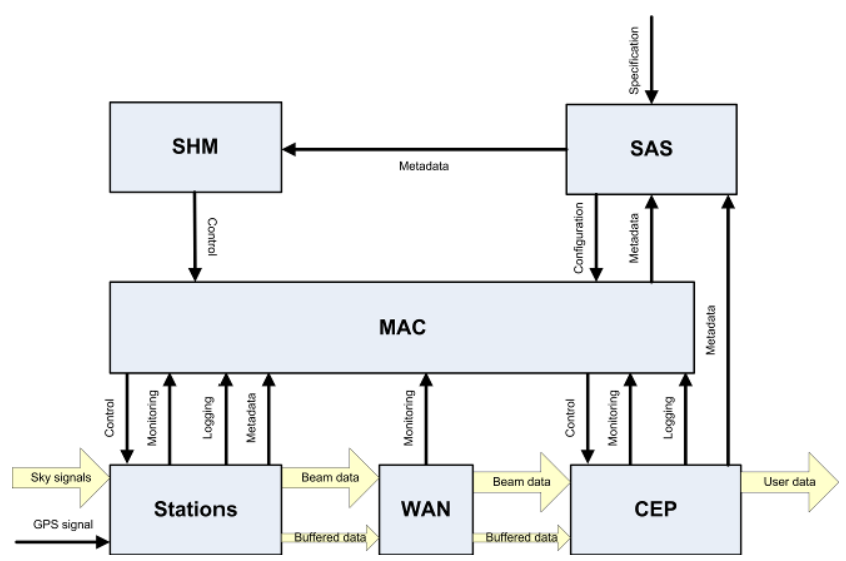

Figure 6 - LOFAR system architecture

Wide Area Network (WAN)-Since the stations are distributed over a large physical area a wide area network is required to connect all those stations. For the signals the WAN subsystem acts as a transparent layer. As shown in Figure 5 all data from the stations needs to come together in the central systems. Hence all data have to be sent to one central location. This was also one of the reasons to position the stations in arms (see Figure 2), which allows us to economically connect the stations with a fiber infrastructure. All fiber connections terminate in the so called concentrator node in the heart of LOFAR. There the signals are re-routed to the central processing (CEP) systems in Groningen.

Central Processor (CEP)-The central systems are responsible to combine the beam data from all stations. There are several observational modes, each requiring a different way of combining the stations. In the aperture synthesis mode for imaging applications the stations are correlated with each other, while in the tied array mode the stations are added to each other. The central processing systems are also responsible to reduce the data further dependent on the observation at hand.

Scheduling, Administration and Specification (SAS)-The instrument control software components fulfill the major task to execute observations on the complete (distributed) instrument. The life cycle of an observation starts in the proposal and specification tools and ends when all data products are archived and exported, from which time onwards the observation data may have a continued life in data archives/database systems.

Monitoring and control (MAC) - After the specification of an observation, the instrument usage is scheduled, resulting in a schedule that is handed over to the control and monitoring package. At the specified time, the measurements will be executed on the specified subsystems of the instrument, in general resulting in a raw dataset being stored at the central storage. The main responsibility of MAC is to control the system (in real-time) based upon the actual configuration of that moment. Additionally, MAC 
facilitates the (real-time) monitoring of the present state of the system. A real-time distributed database is used for MAC.

System Health Management (SHM) - The system health management subsystem continuously analyses the performance of the instrument and updates the resource availability databases used by the scheduler and monitoring and control packages. The main functions of SHM will be the early detection of system failure, the accurate identification of failing components and the support for remaining actions. The input for this is generated at various stages in the system in parallel with the normal data stream.

\section{Functional Decomposition of the System}

In this section the decomposition of the required functionalities over the main subsystems is explained. One of the general rules that are applied is that the amount of hardware in the field is minimized, since maintenance and operational costs of equipment in the field is much higher than equipment at the central location.

The functional signal flow is depicted in Figure 7. The signal flows from left to right, starting off with the antenna. One of the main functions of the receiver is to amplify and select the frequency of the signal, preserving the signal to noise ratio required. Also, the conversion of the analog signal into a digital signal is part of the receiver.

In interferometry it is important to keep the signal paths equal in (electrical) characteristics (because in fact differences between signals received are measured). This also applies to the signals before beamforming. Any difference in gain or phase introduced prior to the beamforming operation will degrade the signal to noise ratio (here defining "signal" as the signal of interest, the sky noise, and the "noise" as the noise generated by the system). For these reasons early sampling and digitization is preferred and therefore placed before the beamformer in the design shown in Figure 7. In the beamformer, the first spatial selection is made by applying time differences between the receptors in the station field and subsequently summing those signals.

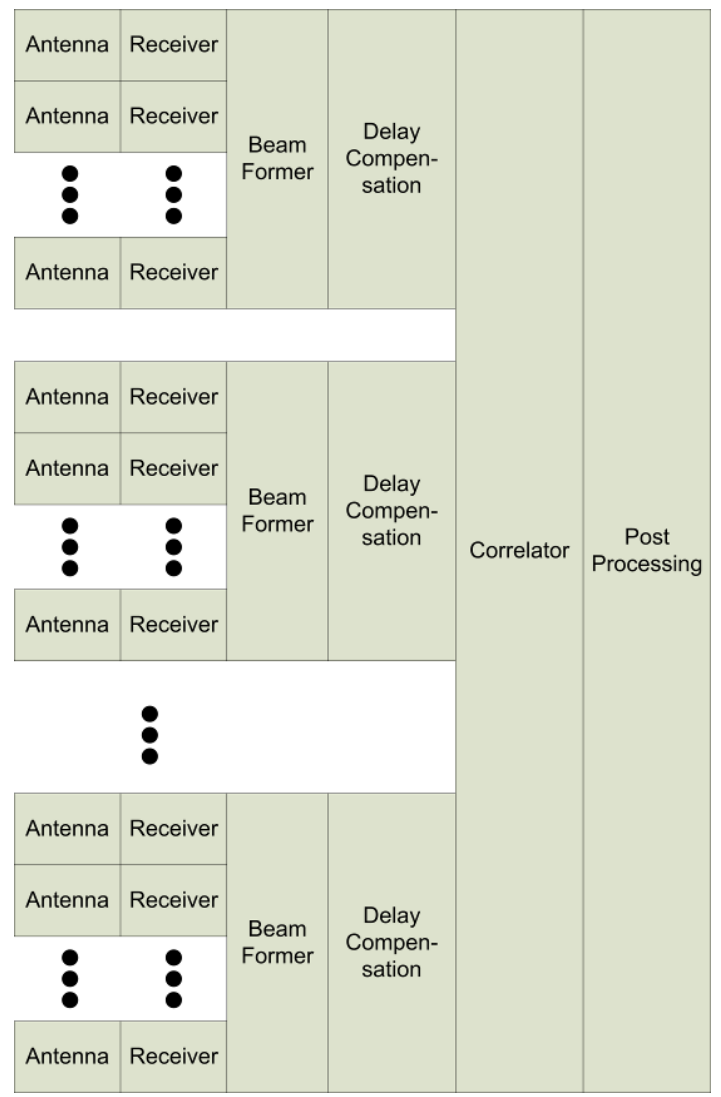

Figure 7 - Global functional LOFAR data path

The rest of the processing steps take place in the CEP subsystem (Figure 6). A second compensation for time differences between the signals from the station fields is applied prior to correlation by phasing up the station signals for a certain observation direction (second step of making spatial selectivity). In the correlator the station signals are combined and correlated with each other, resulting in the full correlation matrix. In the post processing block all "after correlation" operations are assigned to, i.e. calibration and imaging.

Beamformer - The antennas in the station form a phased array, producing one or many station beams on the sky. Multi-beaming is a major advantage of the phased array concept. It is not only used to increase observational efficiency, but may be vital for calibration purposes.

To form a phased array at station level, the analog antenna signals must be delayed and added to form a beam on the sky. Moreover the beamformer should be able to track sources on the sky and be flexible in exchanging beams for bandwidth.

The beamformer can be implemented by using true time delays or by applying phase shifts on "narrow" subbands. For the last solution an error is made at the edges of each subband (see Figure 8), since the phase is frequency dependent and only one phase can be set per subband. 

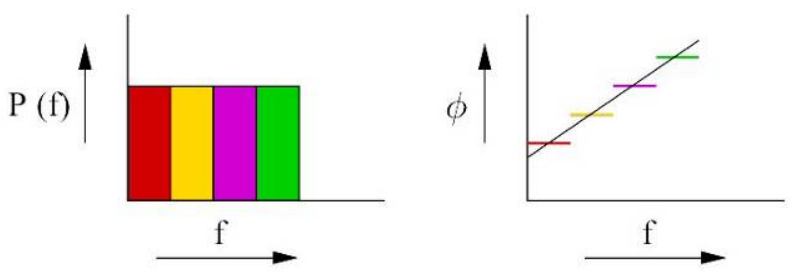

Figure 8 - Illustration of 4 subbands and the error which is introduced by approximating the time delays by phase shifts per subband (the black continuous line on the right hand side is the ideal phase)

Since for the correlator architecture a certain frequency resolution is required and for the beamformer a certain (other) frequency resolution is required as well (if implemented by phase shifts), it was decided to implement the station beamforming with phase shifts after a first stage filterbank which realizes a frequency resolution sufficient for the beamforming operation. A second stage filterbank will make an even higher frequency resolution which is required before the correlator. Another reason to split the filter banks up is that the first stage filter bank is more expensive than the second stage filter bank because the first stage filter bank operates per antenna while the second stage filter bank operates on beams. Since no extra significant data reduction will be done after the second stage filterbank, that functionality will be implemented in the central systems.

Correlator-In LOFAR a FX correlator (first Fourier transform and then correlating the resulting channels) is implemented. Since only a Fourier transform in the FX correlator leads to a significant amount of leakage between the channels filterbanks are used prior to the correlator. This architecture is also known as HFX (Hybrid FX correlator) architecture.

\section{Station ArChitecture}

The main function of the stations is to receive sky signals and select from those the specific signals of interest in time, frequency and spatial domain. This results in a reduction of data which was originally received by the station system [5].

\section{Functional Decomposition}

The signal block diagram of the station is shown in Figure 9. After the receiver a filterbank divides the band in subbands. For each subband an individual beamformer is used to make the spatial selectivity of a station.

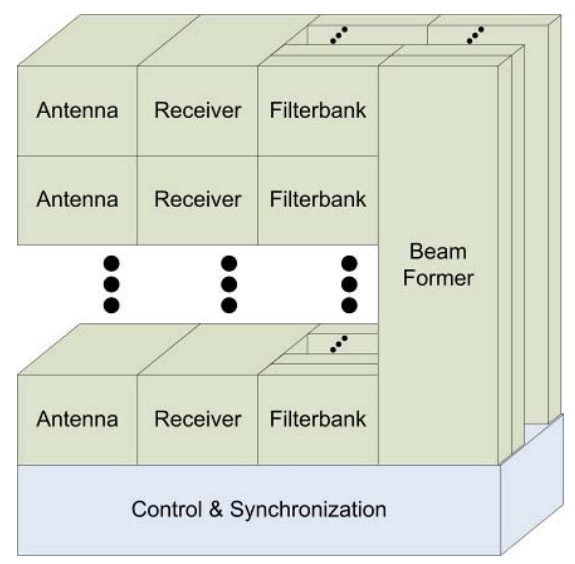

Figure 9 - The signal block diagram of the station

\section{The Antennas}

To achieve an effective area of order 1000 square meters, the antennas within a station are spread over a field of $\sim 85$ meter in diameter. All those antenna signals must be brought together in the beamformer to create a phased array. To reduce Electro Magnetic Interference (EMI) the choice was made not to do the sampling and digitization of the antenna signals near the antenna. Hence, the A/D conversion will take place in a central housing near the station for all antennas. The analog antenna signals will be transported via coax cables to the housing. So, the antennas are localized throughout the field, while the rest of the station hardware is localized at a central place for each station field.

The operating frequency range of LOFAR is from $10 \mathrm{MHz}$ to $240 \mathrm{MHz}$, while the antennas are being optimized for the range $30-80 \mathrm{MHz}$ and 120-240 $\mathrm{MHz}$. Since, the optimized bandwidth of the operating frequency range spans 8 octaves at least two types of antennas are necessary in order to fulfill the sensitivity requirements. Therefore, two types of antennas are developed: the Low Band Antenna (LBA) and the High Band Antenna (HBA). To accommodate science below $30 \mathrm{MHz}$ an extra provision is made for a third antenna, also referred to as the Low Band Low (LBL) antenna. In that context the LBA is also referred to as Low Band High (LBH) antenna.

Each HBA antenna tile is composed of 16 antenna elements. By analog beamforming these antenna elements are combined at tile level.

Since there are no requirements set to observe in the low band mode and in the high band mode instantaneously, all three antennas share the same receiver. In the receiver a switch is made between the antennas.

A picture of both antenna types is shown in Figure 10. 


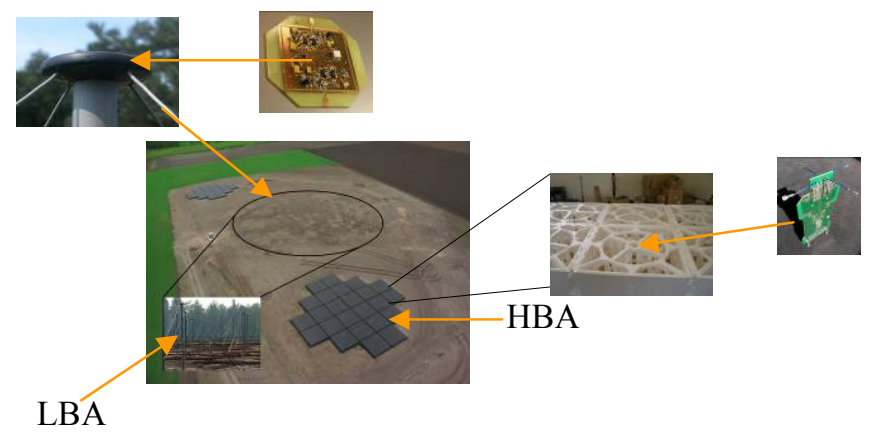

Figure 10 - Photographs of antenna hardware in a station

\section{The Receiver System}

In the receiver the antenna signals must be converted to base band, amplified, filtered and converted to the digital domain. Among a variety of receiver architectures which can be used, a sub-sampling architecture is chosen because:

- No mixers are required (mixers suffer from reciprocal mixing [6] which is degrading the receiver sensitivity if strong interferers are present)

- No extra Local Oscillator signal distribution is required

The counterpart of the advantages is that

- The A/D converter must have a higher analog bandwidth

- Multiple bandpass filters should be implemented to select the frequency band

The maximal bandwidth is $48 \mathrm{MHz}$, while the LBA band runs from $30-80 \mathrm{MHz}$. Selecting a band from the LBA band requires a cut off region for the filters as well and therefore it is chosen to convert the whole LBA band at once. Then, after digital filtering a band of $48 \mathrm{MHz}$ can be selected. Since observing in the FM band is useless a sample frequency of $200 \mathrm{MHz}$ is chosen, which results in a Nyquist edge almost in the centre of the FM band. Three usable Nyquist zones can be used in this way, as depicted in the lower bar of Figure 11.

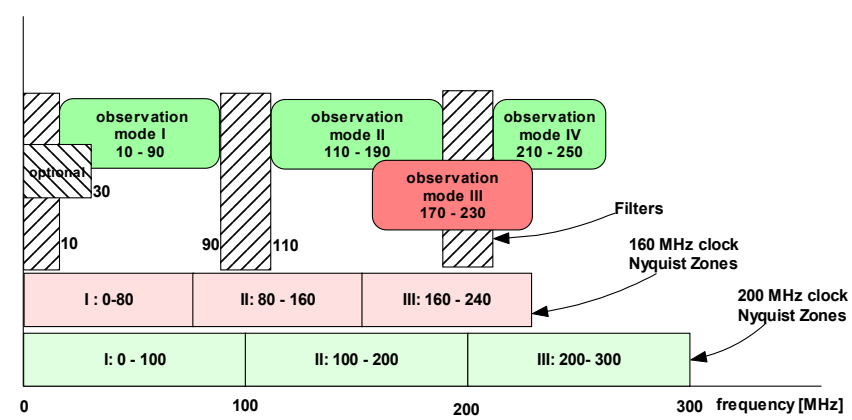

Figure 11 - Observation modes in the station receiver

At the edges of the Nyquist zones aliasing will occur due to the cut of region of the analog filters. Hence, the areas around the edges of the Nyquist zones suffer from loss in sensitivity. For that reason an alternative sampling frequency of $160 \mathrm{MHz}$ will be accommodated. That results in the Nyquist zones shown in the upper bar of Figure 11. The frequencies around $200 \mathrm{MHz}$ can be observed with the
$160 \mathrm{MHz}$ sampling frequency.

\section{Station Digital Processing}

Filterbank-As is explained in Section 3 the function of the filterbank is to make the spectral resolution for the correlator. This spectral resolution can be used for the beamformer as well to apply phase shifts instead of true time delays for all the antenna signals. By approximating the true time delays by phase shifts in a limited frequency band an error is made since only one phase can be set per subband. This causes decorrelation at the subband edges. From this perspective the subbands must be as small as possible. However, from a cost perspective the total number of subbands should be limited. In the final design the complete sampled band is split in 512 subbands resulting in a maximal subband width of about $195 \mathrm{kHz}$. With a selector in between the filterbank and beamformer a subset of the total bandwidth can be selected. The maximal number of subbands to be chosen is 248 . These can be selected randomly over the band.

Beamformer-The beamformer in a station will phase up the antenna signals for a certain direction and tracks a source. The update rate of the beamformer is set to 1 second, so a standard computer can be used to calculate the beamformer weights and send that to the hardware.

Calibration-In Section 3 was already mentioned that differences in gain or phase introduced prior to the beamforming operation will degrade the signal to noise ratio. In order to minimize the differences all analog signal paths in a station are calibrated because temperature variations of the environment cause gain and phase drifts in the signal paths. Furthermore production tolerances results in static gain and phase differences between the signal paths. The complex beamformer weights applied to each antenna signal and for each subband are an excellent way to be used for the calibration [7] as well. In order to calibrate all signal paths a full cross correlation matrix over all antennas can be calculated for one subband integrated over one second. This information is input for the calibration algorithm which runs on the station control computer. A full bandwidth calibration can be done by hopping through the band, taking each second another subband. Approximately 10 minutes are required to go through the full $100 \mathrm{MHz}$ or $80 \mathrm{MHz}$ band.

Buffering-To enable the science cases for the cosmic rays and transients, a transient buffer is included in the station design. With this buffer one second of raw data can be recorded in a circular buffer for all antenna inputs of a station. This means that the full Nyquist band is stored. The storage time can be increased by exchanging bandwidth for storage time and/or antennas for storage time. Also facilities are required to detect nanosecond effects like cosmic rays and lightning. For these applications a detection algorithm is implemented in front of the buffering. This detection algorithm will act on the raw antenna data. If an event is 
detected a message will be send to MAC. There a decision will be made depending on how many events were detected by the individual dipoles to freeze the circular buffer. After the detection the stored data can be sent to the central system upon request.

\section{Implementation}

The station electronics is integrated in subracks. One side of the subracks contains the receivers. These are via a backplane connected to the digital electronics. Figure 12 shows the digital side of the subrack including all boards.

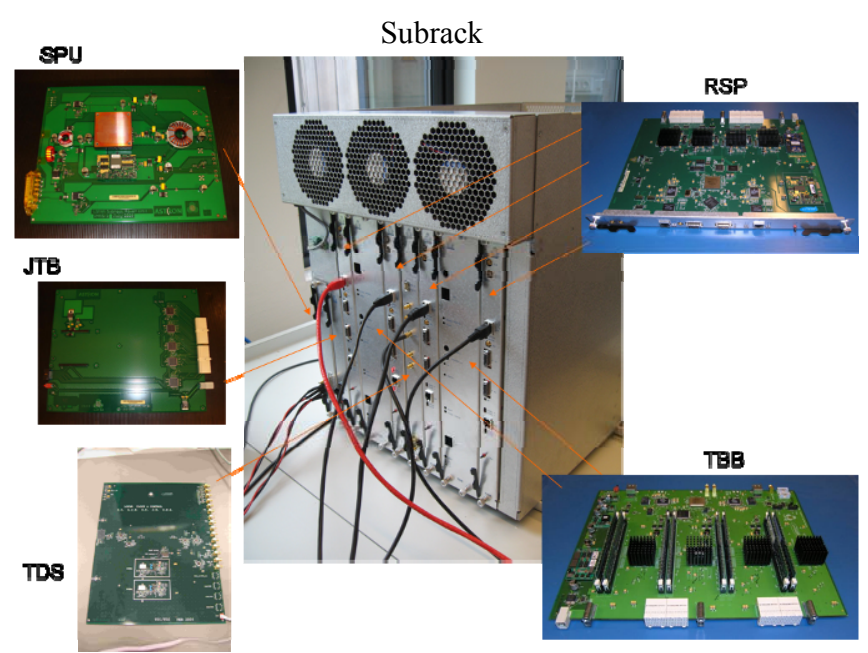

Figure 12 - Showing the internals of a subrack at the digital side (SPU $=$ Station Power Unit, JTB $=$ JTAG Test Board, TDS=Time Distribution System, RSP=Remote Station Processing board, TBB=Transient Buffer Board)

\section{Central Systems ArChitecture}

The main responsibility for CEP is to combine the station data and deliver a data product which can be further processed by the user. The Central Processing Facility is divided in three sections: an on-line section for processing of real-time data streams from the stations, a storage section collecting the processed data streams and making the resulting datasets available to the third section: the off-line processing. This is depicted in Figure 13.

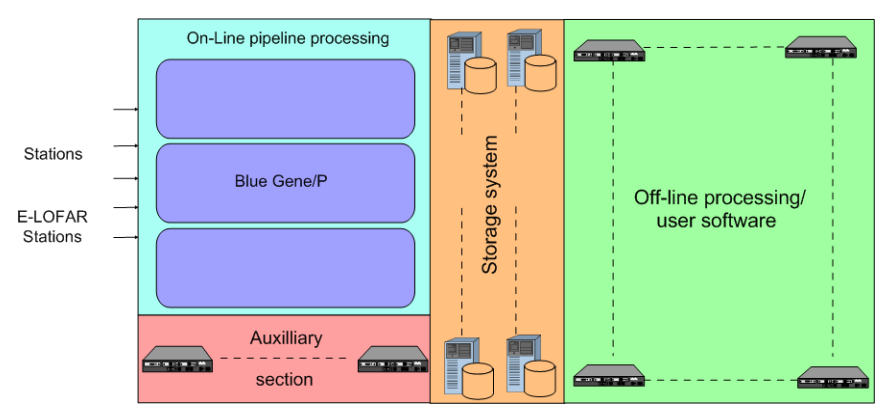

Figure 13 - Central processing systems

The on-line processing section consists of two Blue Gene/P $(\mathrm{BG} / \mathrm{P})$ racks, where the data of the stations are correlated (or beamformed) and subsequently reduced [8]. The resulting data streams from the on-line processing sections are collected in the temporary storage subsystem. The BG/P section contains the connections to the stations (through the WAN). The data sent by the stations are sent in logical packages, each containing a time-frequency window of a single voltage beam. Each IO node of the BG/P will receive data from the stations and run a data handling application that will buffer the input data and synchronize its output stream with the other IO nodes based on the timestamps contained in the data. Large amounts of processing power and internal interconnection bandwidth are provided through the BG/P supercomputer; a peak processing power of 28 TFlops is available for the processing tasks. This processing power in combination with the IO capabilities of $\mathrm{BG} / \mathrm{P}$ allows for a correlator capable to correlate all LOFAR baselines for the full $48 \mathrm{MHz}$ bandwidth together with the channel filterbank.

The storage system provides disk space of $\sim 2$ PByte for the collection of data streams and storage of complete observation datasets for off-line processing. This storage is intended for temporary usage (typically 5 days) until the final data products are generated and archived or the raw data itself is exported or archived.

Finally a general purpose Linux cluster is used for the offline processing. The off-line processing section offers general-purpose processing power and high bandwidth interconnections to the off-line processing applications. Typical applications ran on the off-line cluster are flagging the data, integrating the data in time and/or frequency, subtraction of the main sources, self-calibration [9] and imaging.

Additional off-line processing power will be available in GRID networks, thus potentially providing extra processing power from remote sites. GRID networks also provide the basic infrastructure for data access and data export to user groups. At the end of the pipeline, an archiving system will be available for long term storage or exporting the data.

\section{Status}

The current status at June 2010 is that 6 International stations, 9 remote stations and 20 core stations have been built. Currently 26 stations are used in an operational mode. Already with this amount of stations science can be done with the instrument. In Figure 14 a field centered on the nearby galaxy M51 is shown as observed with LOFAR at frequencies between $120-150 \mathrm{MHz}$ [10]. 


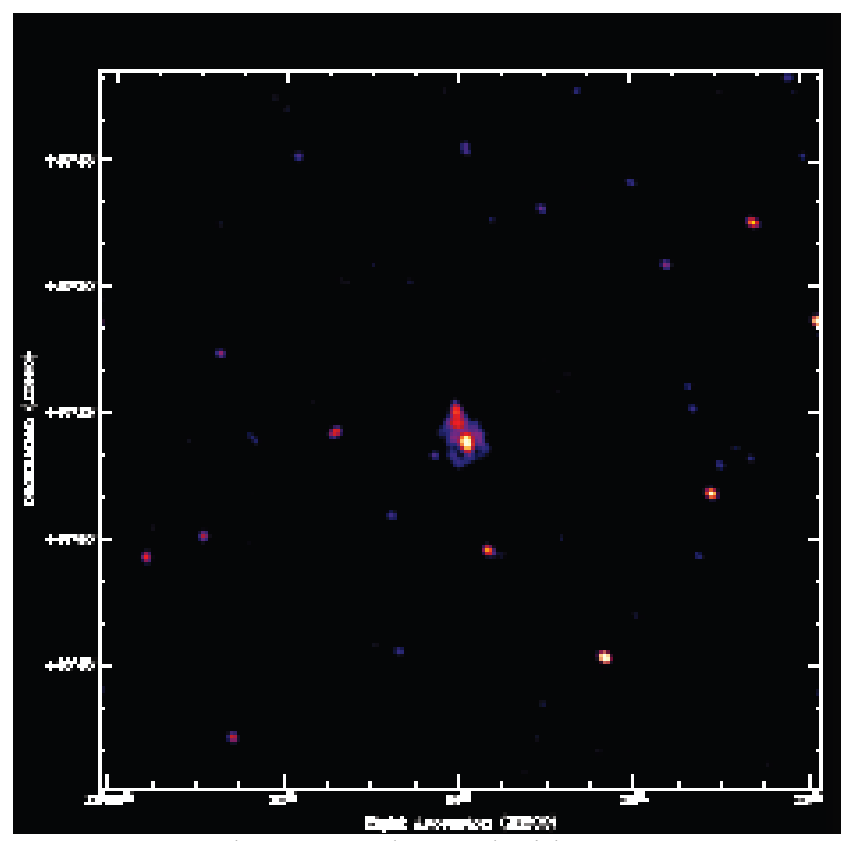

Figure 14 - Galaxy M51 observed with LOFAR [10]

\section{ConCLuSIONS}

In the Netherlands the LOFAR radio telescope is developed and built. Unlike traditional telescopes, the LOFAR telescope is based on phased array principles. In this paper the system architecture is discussed. Currently LOFAR rapidly proceeds to its completion. LOFAR will open a nearly undiscovered window on the lower frequencies and will generate lots of scientific results in areas unexplored so far.

\section{ACKNOWLEDGEMENT}

We like to thank all people who contribute to the LOFAR project. LOFAR is being funded by the Dutch government in the BSIK program for interdisciplinary research for improvements of the knowledge infrastructure. Additional funding is being provided by the European Union, European Regional Development Fund (EFRO) and by the "Samenwerkingsverband Noord-Nederland", EZ/ KOMPAS.

\section{REFERENCES}

[1] J. D. Bregman, Concept design for a low frequency array, Proceedings of SPIE, vol. 4015, 19-33, 2000.

[2] Marco de Vos, Andre W. Gunst, Ronald Nijboer, The LOFAR Telescope: System Architecture and Signal Processing, Proceedings of the IEEE, Vol. 97, No. 8, 14311437, August 2009.

[3] W. A. van Cappellen, S. J. Wijnholds, and J. D. Bregman, Sparse antenna array configurations in large aperture synthesis radio telescopes, Proceedings of the 3rd European Radar Conference, September 2006.

[4] M.J. Bentum, A.W. Gunst and A.J. Boonstra, LOw Frequency Array (LOFAR) - Potential and Challenges, book chapter in Applied Signal and Image Processing: Multidisciplinary Advancements, IGI publishers, 2010.

[5] A.W. Gunst and M.J. Bentum, Signal Processing Aspects of the Low Frequency Array, IEEE International Conference on Signal Processing and Communications, 600-603, 24-27 November 2007, Dubai, United Arab Emirates.

[6] B. Razavi, RF Microelectronics, Prentice Hall, Upper Saddle River, NJ, 1998.

[7] Stefan J. Wijnholds and Alle-Jan van der Veen, Multisources Self-Calibration for Sensor Arrays, IEEE Transactions on Signal Processing, V57, no. 9, 3512-3522, September 2009.

[8] John W. Romein, P. Chris Broekema, Jan David Mol, Rob V. van Nieuwpoort, ACM Symposium on Principles and Practice of Parallel Programming (PPoPP'10), 169-178, January 2010.

[9] Stefan J. Wijnholds, Sebastiaan van der Tol, Ronald Nijboer and Alle-Jan van der Veen, Calibration Challenges for Future Radio Telescopes, IEEE Signal Processing Magazine, V27, no. 1, 30-42, January 2010.

[10] George Heald, et. al., LOFAR paper to be published. 